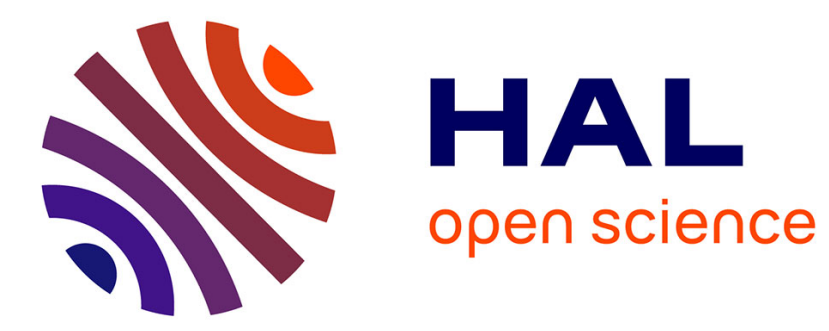

\title{
Wavefront control of a multicore ytterbium-doped pulse fiber amplifier by digital holography
}

\author{
Mathieu Paurisse, Marc Hanna, Frédéric Druon, Patrick Georges
}

\section{To cite this version:}

Mathieu Paurisse, Marc Hanna, Frédéric Druon, Patrick Georges. Wavefront control of a multicore ytterbium-doped pulse fiber amplifier by digital holography. Optics Letters, 2010, 35 (9), pp.1428 1430. hal-00519936

\section{HAL Id: hal-00519936 https://hal.science/hal-00519936}

Submitted on 30 Mar 2012

HAL is a multi-disciplinary open access archive for the deposit and dissemination of scientific research documents, whether they are published or not. The documents may come from teaching and research institutions in France or abroad, or from public or private research centers.
L'archive ouverte pluridisciplinaire HAL, est destinée au dépôt et à la diffusion de documents scientifiques de niveau recherche, publiés ou non, émanant des établissements d'enseignement et de recherche français ou étrangers, des laboratoires publics ou privés. 


\title{
Wavefront control of a multicore ytterbium-doped pulse fiber amplifier by digital holography
}

\author{
Mathieu Paurisse, ${ }^{*}$ Marc Hanna, Frédéric Druon, and Patrick Georges \\ Laboratoire Charles Fabry de l'Institut d'Optique, Campus Polytechnique, RD 128, 91127 Palaiseau Cedex, France \\ *Corresponding author: Mathieu.paurisse@institutoptique.fr
}

Received February 11, 2010; accepted March 15, 2010; posted March 29, 2010 (Doc. ID 124195); published April 28, 2010

We demonstrate the active wavefront control of a 19-core ytterbium-doped fiber amplifier by the digital holography technique. The output wavefront is corrected even in the presence of large externally induced perturbations, with a measured output Strehl ratio of 0.6 in all cases. The wavefront-controlled multicore fiber is used to amplify $800 \mathrm{ps}$ pulses at $1064 \mathrm{~nm}$ at a repetition rate of $40 \mathrm{kHz}$, and a gain of $23 \mathrm{~dB}$ is obtained. (C) 2010 Optical Society of America

OCIS codes: $060.2320,090.1995,070.6120$.

Fiber lasers have attracted significant interest because of their advantages in terms of efficiency, compactness, reliability, and thermal behavior. However, in the pulsed regime, the output power of such devices is limited by nonlinear effects and material damage. Increasing the effective area of the beam propagating inside the fiber is desirable, but this technique is limited because of multimode issues and beam degradation for large transverse dimensions. A widely studied alternative is the coherent combination of fiber lasers or amplifiers [1,2]. To increase considerably the effective area, a large number of emitters is needed, but the system becomes more complex and more sensitive to misalignments between the emitters, and a high fill-factor is difficult to maintain, resulting in a decrease of the energy in the central lobe of the far field. An interesting alternative is to apply this concept to an integrated architecture, such as multimode or multicore fibers.

In multimode fibers, the technique consists in locking the modes propagating inside the fiber in phase at the output of the fiber. Wavefront control by digital holography [3] has been recently demonstrated in a passive fiber supporting few modes [4]. However, the use of a multimode fiber with this technique is limited for two reasons. First, it is difficult to fabricate highly multimode polarization-maintaining (PM) structures. Second, the gain in the fiber and the velocity group dispersion are mode dependent, and the phase-conjugation system cannot compensate for this. For these reasons, it is interesting to study another structure that is less gain dependent and that can be PM with a high effective area.

In this sense, multicore fibers (MCFs) are very promising in terms of effective area, and can be PM. Another advantage is that the emitters are all mechanically and thermally strongly coupled, which make the system less sensitive to global phase variations [5]. The use of Yb-doped MCFs has been demonstrated in several laser configurations [6]. Fundamental mode operation of a 19-core $\mathrm{MCF}$ amplifier has also been demonstrated [7] without wavefront control. However, this operating regime depends strongly on the injection conditions. It is therefore in- teresting to investigate alternative methods using wavefront control.

In this Letter, we demonstrate the wavefront control of a 19-core $\mathrm{Yb}$-doped MCF amplifier using a digital holography technique. This technique relies on the use of the phase-conjugated -1-order of diffraction of a dynamic holographic diffraction pattern recorded on a liquid-crystal-on-silicon spatial light modulator (SLM) (resolution $1920 \times 1080$ and frame rate $60 \mathrm{~Hz}$ ). The architecture, along with technical details of the experimental setup, have been described in our previous work in the context of largemode-area (LMA) fibers [4]. The cores of the fiber used in this work have a diameter of $7 \mu \mathrm{m}$ and a pitch of $11 \mu \mathrm{m}$, which makes them evanescently coupled. As a consequence, the global fiber structure supports several modes, called supermodes. Although the fiber is not PM, the depolarization measured over $5 \mathrm{~m}$ is very low: a linear polarization can be experimentally recovered at the output of the fiber with a quarter-wave plate. A polarization extinction ratio of 93\% is measured, even when the fiber is pumped. The experimental setup is shown in Fig. 1. A laser source at $1064 \mathrm{~nm}$ is separated into probe and reference beams. The probe beam is coupled to the MCF. The fiber is pumped by a $26 \mathrm{~W}$ fiber coupled laser diode emitting at $976 \mathrm{~nm}$. The CCD 1 images the distorted beam at the output MCF facet and makes it interfere with the reference beam. The pattern is displayed continuously on the SLM at a frame rate of $12 \mathrm{~Hz}$

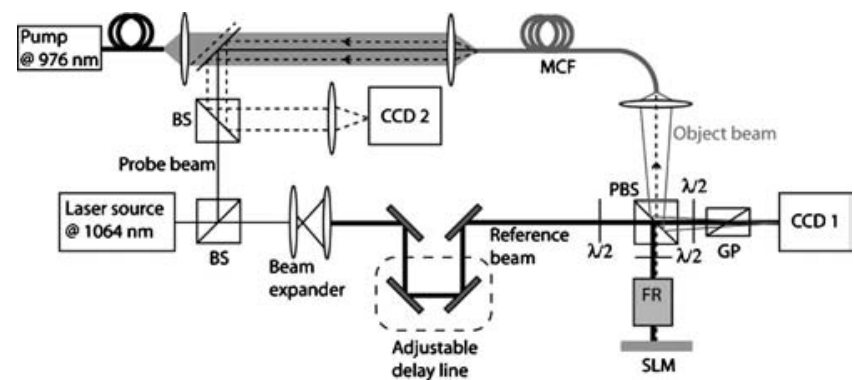

Fig. 1. Experimental setup. BS, beam splitter; PBS, polarizing beam splitter; FR, Faraday rotator; GP, Glan polarizer. 
(limited by our camera), creating a dynamic holographic diffraction pattern. A fraction of the reference beam with an intensity lower than the damage threshold of the SLM $\left(2 \mathrm{~W} / \mathrm{cm}^{2}\right)$ is sent to the modulator via the PBS and is diffracted to create the phase conjugate of the object beam, the seed pulse. The diffraction efficiency (in the -1 -order) is about $10 \%$. The seed is coupled into the fiber, and while it propagates back through it, the spatial phase distortion due to the propagation in the fiber is compensated by the SLM-induced phase conjugation. At the output of the fiber, a corrected beam (in dotted lines) with a plane wavefront is obtained and analyzed on CCD 2.

The hologram recorded on CCD 1 gives access to the spatial phase of the object beam. We recorded three holograms with no pump power, $12 \mathrm{~W}$, and $26 \mathrm{~W}$ pump power (Fig. 2). Two phase vortices can be seen on the holograms. This shows that the wavefront of the object beam is not flat, and consequently that it differs from the fundamental supermode of the MCF: the probe beam at the output is composed of several supermodes that interfere, leading to this complex phase profile. In addition, it can be seen that the three holograms are identical, which indicates that there is no self-organization of the object beam due to the presence of gain. This shows that an external control of the wavefront of the beam exiting the fiber is necessary.

To verify that we were able to generate a corrected beam with a plane wavefront, we first used a longitudinally single-mode Nd:YAG cw laser. Its narrow spectral linewidth $(<1 \mathrm{kHz})$ allows interference between the output corrected beam and the reference beam with a plane wavefront on CCD 2 without delay line. Figure 3(a) shows the interference pattern when the active wavefront correction is working. The interference fringes are all parallel and equally spaced, indicating that the output beam has a plane wavefront. The spatial phase of the output beam can be degraded by turning off the active correction and applying a pressure on the fiber. This modifies the relative phase between the cores inside the fiber and the beam is no longer corrected. The effect is shown on Fig. 3(b). A strong phase distortion appears on the corrected beam, leading to the creation of phase vortices. When the active correction is turned on again, the interference pattern switches back to its original profile [Fig. 3(c)] without phase vortex. An advantage of digital holography is that no wavefront reconstruction is needed, since it is automatically performed by the holographic diffraction pattern. This allows the correction of complex phase distortion such as phase

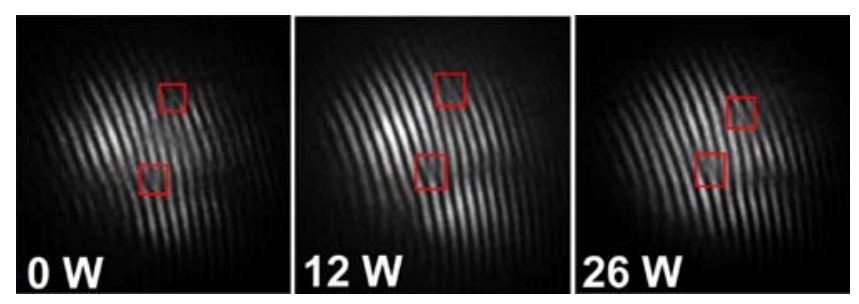

Fig. 2. (Color online) Hologram recorded on CCD1 for 0 , 12 , and $26 \mathrm{~W}$ pump power. The regions of interest (phase vortices) are indicated in the small squares (red online).

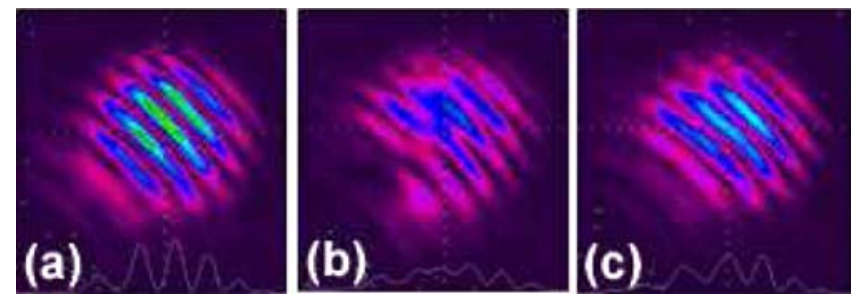

Fig. 3. (Color online) Interference fringes between corrected and reference beams. (a) Active correction $\mathrm{ON}$, no pressure applied. (b) Active correction OFF, pressure applied. (c) Active correction ON, pressure applied.

vortices.

As the limitations of fiber amplifiers mainly arise with high peak powers, it is important to demonstrate the feasibility of digital holography in the pulsed regime. This experiment was performed with $800 \mathrm{ps}$ pulses at a repetition rate of $40 \mathrm{kHz}$ at $1064 \mathrm{~nm}$. In this temporal regime, the group-delay dispersion between supermodes is low compared to the pulse duration and does not affect the phase correction. We checked the efficiency of the wavefront correction by observing simultaneously the near field and the far field of the corrected beam, and by evaluating the Strehl ratio (SR). The SR is evaluated on the far field. A theoretical far field is evaluated by calculating the Fourier transform of the square root of the near-field intensity, therefore assuming that all the cores are in phase. This theoretical far field is compared to the measured far field and the SR is deduced (Fig. 4). With active correction, we measured an SR of $65 \%$. Without applying a pressure to the fiber, the SR drops down to 29\%, and when the correction is turned on again, SR increases up to $59 \%$. The measured gain inside the fiber is shown in Fig. 5. We obtain a gain of $23 \mathrm{~dB}$ at maximum pump power, corresponding to an average output power of $170 \mathrm{~mW}$. The far-field and near-field intensity profiles are shown versus different pump power: it is clear that the phase correction works over the whole pump
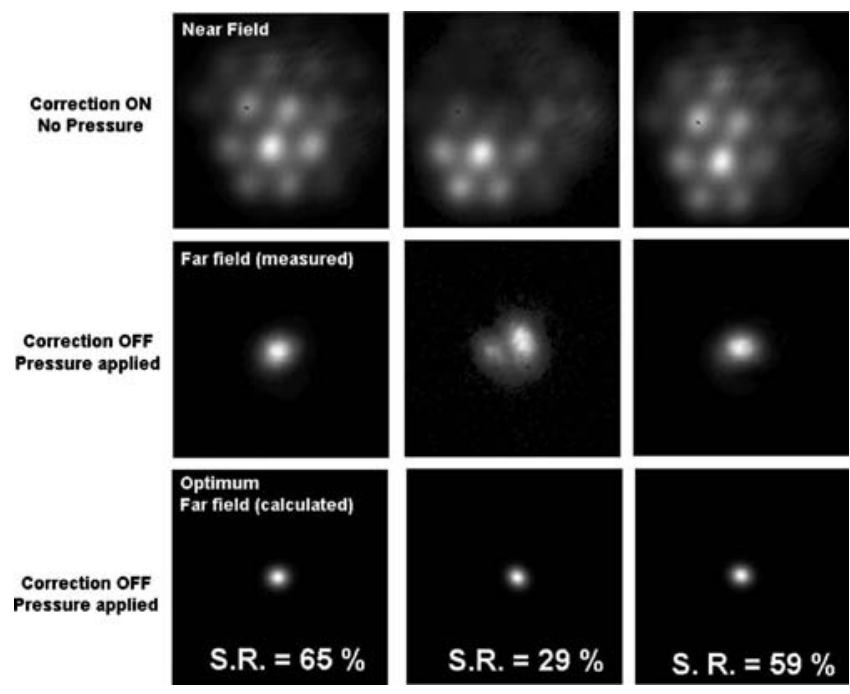

Fig. 4. Measured near fields (top), far fields (middle), and calculated far fields with constant phase (bottom). The images are normalized in intensity. The Strehl ratio is given for each situation. 


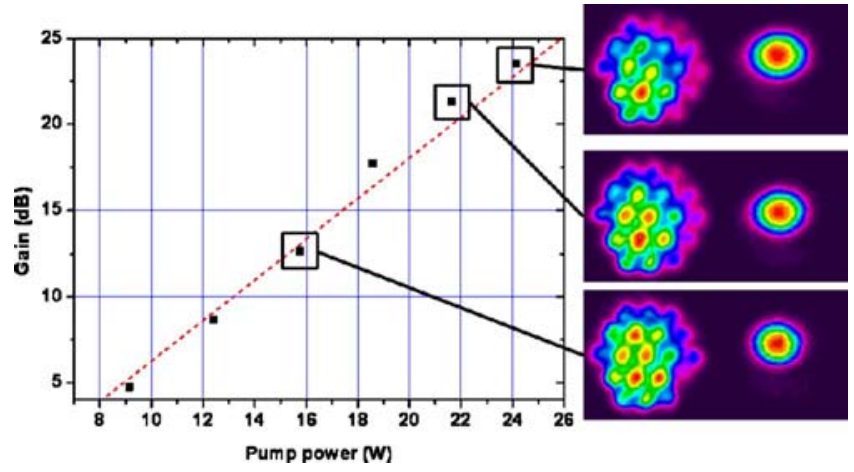

Fig. 5. (Color online) Gain versus pump power and corresponding near fields (left images) and far fields (right images).

range. The near field shows that the corrected beam is slightly off center compared to the central core of the fiber and differs from the fundamental supermode of the fiber. Indeed, the corrected beam exits the fiber with the same characteristics as the probe beam, which means that in our case, the probe beam was not perfectly centered on the central core. However, this is not important for the quality of the corrected beam, as it can be seen on the far field that the beam remains Gaussian.

Like all phase-conjugation methods, the digital holography technique cannot compensate for modedependent gain or loss inside the fiber. For multimode fibers, it is well known that the gain depends on the mode structure propagating inside the fiber [8]. This makes the digital holography technique difficult for multimode fiber amplifiers. Since we demonstrated that this technique works in MCF even with high gain $(23 \mathrm{~dB})$, this indicates the low dependence of the gain with respect to the supermode structure and also the absence of self-organization in MCF amplifiers. This can be understood by noting that, unlike in multimode fibers, both the gain and the supermodes are strongly located in the singlemode doped core areas that have very similar profiles and gain. Therefore, the overlap integral between modes and gain-providing areas does not vary much from supermode to supermode.

In conclusion, we demonstrated wavefront control by digital holography in a 19-core ytterbium doped fiber amplifier in cw and pulsed regimes. Selforganization in the fundamental supermode was not observed, and wavefront correction was demonstrated with a gain up to $23 \mathrm{~dB}$. Our system shows efficient correction of complex phase defects such as phase vortices: the SR was increased by a factor of 2 . The effective area of the MCF is directly proportional to the number of cores and can be scaled easily. Our technique can be used for noncoupled MCF, which can be PM, and thus provides an alternative way to scale the energy of pulsed fiber systems.

The authors thank the Manolia group from the Laboratoire Charles Fabry de l'Institut d'Optique for the loan of the cw longitudinally single-mode laser, and the Agence Nationale de la Recherche program Coherent Amplification Network project for funding. M. Paurisse acknowledges the funding of his Ph.D. by the French Délégation Générale de l'Armement.

\section{References}

1. C. X. Yu, J. E. Kansky, S. E. J. Shaw, D. V. Murphy, and C. Higgs, Electron. Lett. 42, 1024 (2006).

2. E. C. Cheung, M. Weber, and R. R. Rice, in Advanced Solid-State Photonics, OSA Technical Digest Series (Optical Society of America, 2008), paper WA2.

3. C. Bellanger, A. Brignon, J. Colineau, and J. P. Huignard, Opt. Lett. 33, 2937 (2008).

4. M. Paurisse, M. Hanna, F. Druon, P. Georges, C. Bellanger, A. Brignon, and J. P. Huignard, Opt. Express 17, 13000 (2009).

5. I. Hartl, A. Marcinkevicius, H. A. McKay, L. Dong, and M. E. Fermann, Advanced Solid-State Photonics, OSA Technical Digest Series (CD) (Optical Society of America, 2009), paper TuA6.

6. B. M. Shalaby, V. Kermene, D. Pagnoux, A. DesfargesBerthelemot, A. Barthélémy, M. Abdou Ahmed, A. Voss, and T. Graf, Appl. Phys. B 97, 599 (2009).

7. Y. Huo, K. Cheo, and G. King, Opt. Express 12, 6230 (2004).

8. N. Aandermahr and C. Fallnich, Opt. Express 16, 20038 (2008). 